Relations industrielles

Industrial Relations

\title{
Éthier, Gérard, La qualité totale: nouvelle panacée du secteur public?
}

\section{Marcel Laflamme}

Volume 50, numéro 3, 1995

URI : https://id.erudit.org/iderudit/051045ar

DOI : https://doi.org/10.7202/051045ar

Aller au sommaire du numéro

Éditeur(s)

Département des relations industrielles de l'Université Laval

ISSN

0034-379X (imprimé)

1703-8138 (numérique)

Découvrir la revue

Citer ce compte rendu

Laflamme, M. (1995). Compte rendu de [Éthier, Gérard, La qualité totale: nouvelle panacée du secteur public ?]. Relations industrielles / Industrial Relations, 50(3), 670-672. https://doi.org/10.7202/051045ar

Tous droits réservés (C) Département des relations industrielles de l'Université Laval, 1995
Ce document est protégé par la loi sur le droit d'auteur. L'utilisation des services d'Érudit (y compris la reproduction) est assujettie à sa politique d'utilisation que vous pouvez consulter en ligne.

https://apropos.erudit.org/fr/usagers/politique-dutilisation/ 
concerne l'étude des griefs et de l'ensemble du mécanisme de l'arbitrage des griefs. Évidemment, la publication du livre a eu lieu avant que de nouvelles règles législatives ne s'appliquent, notamment, en ce qui a trait à la durée de la convention collective. (À ce sujet, voir Rodrigue Blouin et Fernand Morin, Droit de l'arbitrage de grief, $4^{\mathrm{e}}$ édition, Cowansville, Éditions Yvon Blais, 1994, p. 52.) D'autre part, sous les sous-titres "Convention exclusive " (p. 186) ou " Convention effective et stable" (p. 187), il aurait été pertinent de citer les articles du Code du travail (L.R.Q., c. C-27) faisant référence à ces notions d'exclusivité et de durée de la convention collective. Le même commentaire peut s'appliquer à d'autres endroits (exemple : la sentence est sans appel et lie les parties, p. 332).

Quant aux deux derniers chapitres, l'exposé sur les MRL et leur raison d'être est un point de départ. Certaines données déjà existantes ou à collecter pourraient davantage nous renseigner sur les organisations qui ont mis sur pied des MRL au Québec, au Canada ou dans d'autres pays, actuellement. Quelques exemples sont mentionnés comme Northrup (1946), General Electric, Trans World Airlines et Control Data à partir de publications datant de 1984 (p. 354 368 ), ce qui pourrait être mis à jour ultérieurement.

Par ailleurs, je n'ai pas vérifié toutes les sources de jurisprudence mentionnées par l'auteur dans son analyse. Je présume que celles-ci furent choisies avec soins. Je signale enfin quelques petites corrections de forme. La plus importante concerne les références législatives comme les Chartes des droits individuels, la Charte de la langue française ou le Code civil. À cet effet, des règles sont prévues pour citer les lois et elles n'ont pas été appliquées. En outre, l'usage de certaines expressions sont à revoir comme " monter dans l'entreprise " (p. 63), "lancer un MRL" (p. 389) ou "politiques organisationnelles versus de relations avec les employés" (p. 50). Aussi, la référence de Trisler, 1984 (p. 368) n'est pas mentionnée dans la bibliographie.

Cet ouvrage permet donc de s'initier aux aspects techniques et théoriques de l'étude des mécanismes de résolution des griefs et des plaintes dans les organisations. L'étude de l'arbitrage des griefs occupe une part importante du volume.

HÉLÈNE GASCON

Université du Québec à Rimouski

\section{La qualité totale: nouvelle panacée du secteur public? \\ par Gérard ÉTHIER, Sainte-Foy, Qc, Presses de l'Université du Québec, 1994, 220 p., ISBN 2-7605-0758-0.}

Essentiellement, l'ouvrage décrit et analyse des expériences d'implantation de projets qualité dans cinq organisations publiques, bien ciblées et complémentaires: l'Hôpital du Haut-Richelieu, l'Hôpital Maisonneuve-Rosemont, la ville de Charlesbourg, le ministère de l'Industrie, du Commerce et de la Technologie et la Commission scolaire Saint-Jérôme. La description de ces cas s'effectue en cinq chapitres précédés par une partie intitulée: "Cadre théorique et méthodologique ", le tout complété par un der- nier chapitre ayant trait à la synthèse finale.

Les cas pratiques sont décrits dans un premier temps et sont suivis d'analyses et de commentaires. L'évaluation est surtout d'ordre psychosocial concernant différents paramètres inhérents à la gestion du changement : résistance, approche-client, sentiment d'appartenance, participation, responsabilisation, communication, motivation du personnel, visibilité des gestes, leadership, etc. Cette contribution empirique présentée avec un bon 
style s'avère la partie positive de l'ouvrage apte à être utile pour les théoriciens et praticiens intéressés au phénomène de la qualité totale en secteur public.

La partie faible du travail a trait au premier chapitre comprenant le cadre théorique et méthodologique, et partant, ayant une répercussion sensible sur les commentaires fournis dans l'ensemble de l'œuvre. En effet, les deux sources majeures d'inspiration sur lesquelles est bâtie la recherche renvoient aux huit principes du modèle théorique du Secrétariat de la Fonction publique 2000 et aux cinq principes et cinq actions inhérents au modèle préparé par le Conseil du Trésor de la province de Québec (1987) (p. 20). De ces principes guidant la réforme de l'administration publique, l'auteur dégage les concepts clefs de son étude concernant principalement deux zones majeures d'intervention en qualité totale : 1) les relations clients et 2) la mobilisation du personnel (sentiment d'appartenance, participation, responsabilisation, communication et motivation) (p. 34).

Aucune référence dans l'ouvrage n'est faite aux gourous de la qualité (Deming, Juran, Harrington, Crosby, Feigenbaum, Ishikawa, etc.) ni aux écoles de pensée qui constituent le corpus et le champ d'étude du mouvement de la qualité totale que nous considérons important de préciser ici:

1. L'École de la gestion financière des coûts cachés et de la lutte à toute forme de gaspillage par la recherche des zéro-défaut, zéro-délai, zéro-plainte, zéro-papier et zéro-mépris. Notons que les coûts de non-qualité sont évalués à $20 \%$ du chiffre d'affaires des entreprises privées et à environ $30 \mathrm{mil}$ liards de dollars pour l'ensemble du Québec.

2. L'École qui s'appuie sur le marketing via une stratégie de développement et une culture où l'ensemble de l'organisation est essentiellement centré sur le client et sa raison d'être.
3. L'École du management impliquant une révision radicale des rôles des gestionnaires, des spécialistes et des employés relativement aux dimensions suivantes : plan-qualité, structure-qualité, direction-qualité et contrôle-qualité.

4. L'École des ressources humaines axée sur la mobilisation du personnel, par la formation continue, la généralisation des groupes d'amélioration qualité et l'adoption de systèmes de reconnaissance-qualité.

5- L'École de la théorie des organisations, renvoyant à un nouveau paradigme axé notamment sur la débureaucratisation, l'analyse des dysfonctionnements, le nivellement et l'horizontalisation de la structure pyramidale, la flexibilité et la rapidité des temps de réponse, les relations transversales clients-fournisseurs à l'interne et à l'externe, le décloisonnement des services, l'ajout de la structure ad hoc qualité.

6. L'École de la gestion des opérations et des processus, via la simplification des systèmes, le juste-à-temps, la valeur ajoutée, la démocratisation des données et la réingénierie des processus.

7. L'École de la gestion des approvisionnements et des relations de partenariat avec les fournisseurs externes axées sur les échanges d'expertises, les ententes à moyen terme, la qualification et l'assurance-qualité (ISO9000).

Bref, la référence à la qualité totale comprend fondamentalement ce processus d'amélioration continue de toutes les activités dans le cycle d'opération pour une meilleure réponse au client au moindre coût. Suivant une stratégie intégrée, c'esı la synthèse optimale de tous ces facteurs de progrès. Ainsi le mouvement qualité se veut un concept fédérateur comprenant plus d'une trentaine de pratiques de changements ciblés principalement sur six zones majeures 
d'intervention (M. Laflamme, "La qualité totale: un nouveau paradigme pour les organisations ", Revue Organisation, automne 1994, p. 13-25).

À notre avis, l'auteur n'a pas su bien cerner la spécificité de la qualité totale ; il l'associe à la reprise de thèmes traditionnels (p. 19) et fait une mauvaise distinction entre les concepts de productivité et de qualité (p.16). Nous concluons que l'auteur n'a pas atteint les objectifs (1) et (3) de son étude (p. 29), soit: "Préciser tout le débat actuel sur la qualité des services publics à l'aide des idées et des critiques formulées par certains auteurs et, d'autre part, des modèles théoriques et des concepts qui se dégagent le mieux de tous les écrits. [...] Dégager un ou des modèles généraux pour servir de guides à d'autres organisations qui veulent tendre à un même but. "

Cependant, le second objectif de volume a bien été complété, soit : "à partir de cas réels, décrire et analyser les expériences vécues par certaines organisations publiques qui se sont lancées dans un tel projet". Sur ce point, nous félicitons l'auteur, qui fait oeuvre de pionnier dans ce domaine au Québec et nous souhaitons par cette occasion la publication d'autres ouvrages qui permettent de mieux maîtriser ce phénomène si complexe de la qualité totale.
$\grave{A}$ cet effet, je termine par deux suggestions:

1) Pour les auteurs : retourner aux sources, constituer des équipes multidisciplinaires et aller voir ce qui se passe dans le secteur privé où le mouvement qualité a débuté et a pris une longueur d'avance sur le public (exemples : Xérox et Venmar).

2) Pour les organismes publics : si l'on veut prendre la qualité totale au sérieux, il faut effectuer dans une première phase un diagnostic des coûts cachés de la non-qualité (environ $15 \%$ à $40 \%$ des frais d'exploitation) qui seraient évalués par une firme indépendante (externe). Par la suite, pourrait suivre la démarche d'amélioration continue pour réduire ces coûts cachés à moins de $10 \%$ du coût d'exploitation. Actuellement les consultants en qualité sont embauchés par les gestionnaires-fonctionnaires qui ne prennent naturellement que ce qui leur va dans la panoplie des programmes en qualité totale. C'est pourquoi il est si imprudent de juger le mouvement de la qualité totale à partir d'expériences de courtes durées et implantées de façon fragmentaire.

MARCEL LAFLAMME

Université de Sherbrooke

\section{Immigrés et création d'entreprises: Montréal 1990}

par Denise HELLY et Alberte LEDOYEN, Québec, IQRC, 1994, 305 p., ISBN 289224-191-X.

Le livre que nous proposent Helly et Ledoyen est basé sur un projet de recherche subventionné par la Direction de la recherche du ministère des Communautés culturelles et de l'Immigration du Québec. Il a pour objet d'approfondir les quelques points importants souvent abordés dans la littérature sur l'entrepreneuriat immigré, à savoir les raisons qui poussent les immigrés à entreprendre, le choix du sous-secteur d'activités et l'or- ganisation de la mobilisation des ressources utiles à la création d'une entreprise. Précisons que cette recherche porte exclusivement sur les immigrés entrepreneurs qui s'installent à Montréal, et qu'elle tient davantage compte du nouveau contexte canadien à la suite des changements de conjoncture, voire de structure, économique survenus durant les années 1970 . 